\title{
Smart Bakery: Estudo Prospectivo sobre Internet das Coisas (IoT) Aplicadas ao Setor de Panificação
}

\author{
Smart Bakery: Prospective Study on the Internet of Things (IoT) Applied \\ to the Bakery Sector
}

\author{
Beneildo Rodrigues Oliveira Pereira ${ }^{1}$ \\ Clodoaldo Silva do Nascimento ${ }^{1}$ \\ Jéssica Caroline Rodrigues de Lima ${ }^{1}$ \\ João Paulo Lima Santos ${ }^{1}$ \\ Ramon Cardeal Silva ${ }^{1}$ \\ Silvia Beatriz Beger Uchôa ${ }^{1}$ \\ ${ }^{1}$ Universidade Federal de Alagoas, Maceió, AL, Brasil
}

\begin{abstract}
Resumo
A Internet das Coisas (IoT), solução que permite a conexão entre diferentes objetos por meio da rede mundial de computadores, oferece às empresas de alimentos e bebidas um vasto potencial para otimização de suas operações. Ao mesmo tempo, o setor de panificação e de confeitaria brasileiro é impelido a adotar novas tecnologias, visando ao aumento do seu diferencial competitivo e inovativo. Dentro desse contexto, o presente artigo teve como objetivo realizar a prospecção tecnológica de soluções do sistema IoT com potencial de aplicação no setor de panificação, sendo para isso levantadas as patentes depositadas no Instituto Nacional de Propriedade Industrial (INPI) e no Questel Orbit, assim como as produções científicas registradas nas plataformas Capes e Scielo. A prospecção aponta para um crescimento mundial nas pesquisas e no desenvolvimento de produtos que relacionam IoT e alimentação, principalmente na China. O Brasil, apresenta um cenário incipiente no desenvolvimento dessas tecnologias, embora já existam algumas iniciativas do poder público e da indústria.
\end{abstract}

Palavras-chave: Internet das Coisas. Panificação. Prospecção.

\begin{abstract}
The Internet of Things (IoT), a network that allows the connection between different objects through the world wide web, offers food and beverage companies a vast potential for optimization of their operations. At the same time, the Brazilian bakery and confectionery industry is impelled to adopt new technologies, aiming at increasing its competitive and innovative differential. In this context, the present article aimed to carry out the technological prospection of solutions of the Iot system with potential of application in the bakery sector, for which the patents deposited at Instituto Nacional de Propriedade Industrial (INPI) and Questel Orbit were searched, as well as the scientific productions recorded on the platforms Capes and Scielo. The prospect points to a worldwide growth in the research and development of products that relate IoT and food, mainly in China. Brazil presents an incipient scenario in the development of these technologies, although there are already some initiatives of the public power and industry.
\end{abstract}

Keywords: Internet of Things. Bakery. Prospection.

Área Tecnológica: Propriedade Intelectual. Internet das Coisas. Padarias Inteligentes. 


\section{Introdução}

As mudanças recentes nos processos de produção ocasionadas pelo surgimento da automação e da integração humano-máquina marcam o que se chama de Quarta Revolução Industrial ou Indústria 4.0, movimento que pretende possibilitar maior autonomia na tomada de decisões e ampliar a transparência nas relações máquina e humano (PEREIRA; SIMONETTO, 2018).

$\mathrm{Na}$ base dessa revolução encontra-se a internet das coisas (IoT), estágio avançado da internet em que os objetos, máquinas e equipamentos se relacionam com outros objetos e com seres humanos por meio de dispositivos computacionais capazes de conexão e de comunicação. Nesse sentido, os objetos tendem a assumir o controle de uma série de ações cotidianas, sem haver a necessidade de atenção e de comando por parte dos indivíduos (SANTAELLA et al., 2013). A Revista Forbes (2016) destaca o potencial dessas tecnologias no aperfeiçoamento da experiência de clientes e fornecedores, na medida em que os dados sobre comportamento $e$ experiência dos usuários com os produtos poderão ser enviados diretamente para as empresas responsáveis pela sua produção e comércio, permitindo a construção de perfis de clientes, a quem serão ofertados itens baseados em seu histórico de consumo.

A IoT faz amplo uso das coisas para oferecer serviços para todo tipo de aplicações, por meio da exploração das capacidades, da identificação e captura de dados, e de processamento e comunicação, que reduzem as barreiras entre o mundo físico e o digital, através de dispositivos que se comunicam com os outros, com os data centers e suas nuvens (ZAMBARDA, 2014).

Segundo a Federação das Indústrias do Estado do Rio de Janeiro (FIRJAN), a tendência para as próximas décadas de uma processo produtivo marcado pela "descentralização", no qual a utilização de sensores, sistemas de interconectividade e análise de dados propiciarão a fusão do universo real e virtual na produção (FIRJAN, 2016). No Brasil, leis e normas como a "Lei da Inovação", de 2004; a "Lei da Informática", de 1991; a "Lei do Software", de 1998; o "Marco Civil da Internet", de 2014; e o "Programa Banda Larga para Todos", de 2018, influenciam o investimento em pesquisas e desenvolvimento de tecnologias embarcadas capazes de interagir com diversos ambientes internos ou externos (BIAGGI et al., 2018).

De acordo com levantamento realizado pelo Instituto Tecnológico de Panificação e Confeitaria (ITPC), em parceria com a Associação Brasileira da Indústria de Panificação e Confeitaria (ABIP), o setor de panificação e de confeitaria brasileiro registrou um crescimento nominal de $3,08 \%$ em 2016, atingindo um faturamento de $\mathrm{R} \$ 87,24$ bilhões ao ano e com uma estimativa de geração de emprego de 800 mil pessoas de forma direta e 1,8 milhão de forma indireta. Por outro lado, esse estudo também observou uma queda de 4,06\% no fluxo de clientes das panificações mais tradicionais, ocasionada em parte pela manutenção de estratégias de vendas conservadoras, que não acompanham as novas formas de comunicação da era digital, e pelo crescimento de canais de vendas de menor porte, como conveniências e mercearias locais (SEBRAE, 2016).

Diante desse cenário, as panificações são impelidas a adotar novas tecnologias para gerenciamento, comunicação com clientes, redução de desperdício, visando ao aumento do seu diferencial competitivo e inovativo, de forma a atingir a satisfação de um público mais exigente e seletivo. O sistema em rede da "Internet das coisas" (IoT) oferece às empresas de alimentos e bebidas um vasto potencial para otimizar as operações em diferentes áreas, como rastreabi- 
lidade, conformidade, custo das mercadorias vendidas, tempo de inatividade não planejado, custos de energia, giro de estoque, colaboração entre parceiros, entre outros (BODLA, 2015).

O lançamento do "Plano Nacional da Internet das Coisas" pelo governo brasileiro no fim de 2017 ocorreu como uma tentativa de desenvolvimento sustentável e competitivo da economia brasileira, além de estimulo ao desenvolvimento de tecnologias nacionais, visando a atingir uma diminuição do custo com importações e geração de tecnologia (MINISTÉRIO DA CIÊNCIA, TECNOLOGIA, INOVAÇÕES E COMUNICAÇÕES, 2017).

A partir dessas informações, buscou-se realizar uma prospecção tecnológica de soluções do sistema de rede de Internet das Coisas (IoT) com potencial de aplicação nos meios produtivos do setor de panificação, por meio de buscas de patentes e produções acadêmicas sobre a temática.

\section{Metodologia}

Para realizar a prospecção tecnológica de soluções do sistema IoT com potencial de aplicação no setor de panificação, foi efetuado o levantamento dos processos de pedidos de patentes depositados em âmbito nacional e internacional. Para buscas de pedidos no Brasil, foi utilizada a base do Instituto Nacional de Propriedade Industrial (INPI) e para as buscas internacionais utilizou-se a plataforma Questel Orbit, sistema de busca e análise com informações de patentes de mais de 90 países.

Para a busca de produções acadêmicas sobre a temática foram utilizados o Portal de Periódicos, da Coordenação de Aperfeiçoamento de Pessoal de Nível Superior (CAPES), uma biblioteca virtual que reúne e disponibiliza a instituições de ensino e pesquisa no Brasil o melhor da produção científica internacional e da Scientific Electronic Library Online (SciELO), uma biblioteca eletrônica que abrange uma coleção selecionada de periódicos científicos brasileiros.

É importante salientar que, embora o portal de periódicos Capes seja um sistema gerido por instituição nacional, ele reúne e disponibiliza um acervo de produções científicas nacionais $e$ internacionais, o que torna o número de resultados das buscas realizadas consequentemente maior, não cabendo assim maiores comparações entre os seus resultados e a produção registrada na base Scielo.

Em ambos os processos de busca foram utilizadas palavras-chave em português e em inglês relacionadas aos segmentos de Padaria, Indústria 4.0, Automação e Alimentos, conforme mostra a Figura 1. Visando a obter uma maximização dos resultados, foram aplicados na busca dos termos operadores booleanos e trucagem, como AND, OR e ( ). Todas as pesquisas foram efetuadas nas modalidades avançadas em cada uma das bases consultadas no período que compreende as datas entre 26/05/2018 a 26/06/2018.

A utilização inicial de uma grande variedade de palavras-chave nas bases de patentes e artigos (dez termos de busca) teve por objetivo alcançar o maior número possível de resultados relacionados à tecnologia de IoT e à produção do setor de panificação. Para realização de análise mais detalhada foram escolhidos os termos de busca "bakery OR bread OR pastry OR confectionery OR (bake shop) OR (pastry shop) OR pâtisserie OR padaria OR panificacao" e o termo "Iot AND Food".

A escolha desse primeiro termo de análise teve como intenção obter um panorama geral sobre todas as produções científicas e patentes relacionadas ao setor alimentício de panificação 
desenvolvidas até o momento. A definição e a seleção do segundo termo tiveram como objetivo o levantamento de produções especificas que associassem a Internet das Coisas ao seguimento alimentício.

Para refinar as buscas tecnológicas, foi feita a análise da Classificação Internacional de Patentes (IPC), instrumento que possibilita a organização dos documentos de patente pela área tecnológica a que pertencem, sendo usado com a finalidade de facilitar o acesso às informações tecnológicas e legais neles contidas (MARTINEZ; SANTOS; WINTER, 2015).

Para complementar a pesquisa e apontar uma linha de tendência do interesse pelo público em geral acerca da temática foi realizada análise na ferramenta Google Trends, a qual apresenta o histórico de busca para toda a web em determinado espaço de tempo.

Figura 1 - Metodologia para buscas em bases acadêmicas e de patentes
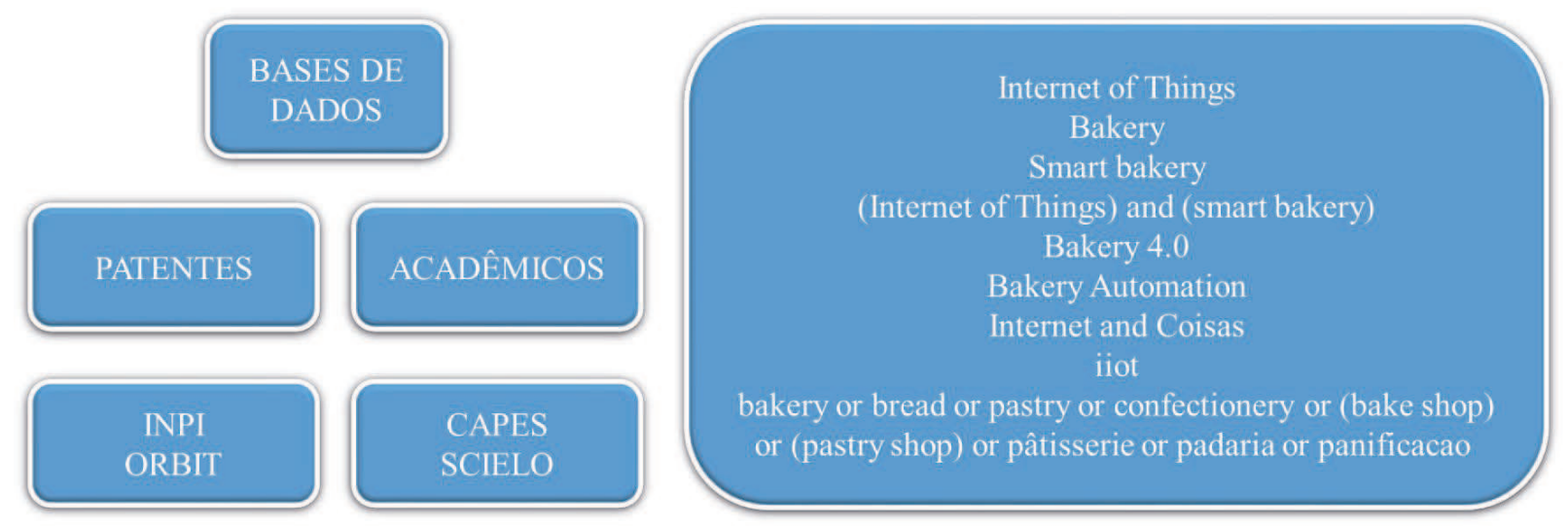

Fonte: Elaborada pelos autores deste artigo (2018)

\section{Resultados e Discussão}

Em relação à busca de periódicos que apresentassem as palavras-chave selecionadas, pode-se dizer que a base da Capes apresentou o maior número de publicações em todas as categorias de pesquisa quando comparados aos resultados obtidos na base Scielo. A base Capes apresentou 447.022 resultados de produções científicas envolvendo termos específicos relacionados ao setor de panificação (bakery or bread or pastry or confectionery or bake shop) or (pastry shop) or pâtisserie or padaria or panificação), enquanto a base Scielo apresentou 788 resultados.

$\mathrm{Na}$ busca realizada na base da Scielo, em cinco categorias de pesquisa foram verificados resultados nulos ("Smart bakery", "(Internet of Things) and (smart bakery)", "Bakery Automation", "Iot", "IoT and Food"), sendo nos demais resultados verificados valores bastante reduzidos, principalmente quando comparados aos resultados obtidos na base Capes.

Em relação à busca de patentes, pode-se dizer a base Orbit apresentou o maior número de patentes envolvendo termos específicos ligados ao setor de padarias, cujo valor foi de 83.904 patentes, enquanto na base nacional do INPI foram encontrados apenas 230 resultados. 
Os resultados observados na base do INPI foram nulos em quatro das dez categorias de pesquisa elencadas ((Internet of Things) and (smart bakery); IoT; Bakery and 4.0; IoT and Food), sendo nas demais categorias de busca observados valores muito reduzidos, sobretudo quando comparados aos resultados verificados na base Orbit.

Por meio dos resultados obtidos nas bases com depósitos de produções nacionais Scielo e INPI, torna-se possível afirmar que o Brasil não investe com expressividade na produção de conhecimento e proteção de tecnologias ligadas ao sistema IoT e ao setor alimentício de panificação. Com isso, o país mantém uma dependência tecnológica, estando posicionado como comprador de tecnologia para a implementação de inovações, o que eleva o custo dos investimentos no setor alimentício de panificação.

Tabela 1 - Palavras-chave utilizadas nas buscas de patentes e periódicos com os respectivos números de documentos encontrados

\begin{tabular}{ccccc} 
& \multicolumn{2}{c}{ PATENTES } & \multicolumn{2}{c}{ PeRiódicos } \\
Palavras-chaves & INPI & Orbit & Capes & Scielo \\
Internet of Things & 3900 & 34819 & 392334 & 56 \\
Bakery & 3 & 11404 & 69669 & 124 \\
Smart bakery & 92 & 1008 & 3209 & 0 \\
(Internet of Things) and (smart bakery) & 0 & 0 & 356 & 0 \\
Bakery Automation & 5 & 923 & 1041 & 0 \\
Internet and Coisas & 32 & 1258 & 2127 & 19 \\
IoT & 0 & 1263 & 2992 & 0 \\
Bakery OR bread OR pastry OR confectionery & & & & 788 \\
OR (bake shop) OR (pastry shop) OR & 230 & 83904 & 447022 & 17 \\
pâtisserie OR padaria OR panificacao & & & & 0
\end{tabular}

Fonte: Adaptada pelos autores deste artigo com dados da base Orbit Intelligence (2018)

O Gráfico 1 apresenta um comparativo entre o número de produções acadêmicas e depósitos de patentes. Percebe-se que a quantidade de artigos se sobrepõe ao número de patentes em termos como "IoT AND Food"; "iiot"; "Bakery AND 4.0" e "Smart Bakery", o que revela a existência de grande interesse científico na junção destes itens e um gargalo tecnológico nestes setores. Já para o termo "Bakery automation" verifica-se similaridade na quantidade de produções científicas e de patentes tecnológicas, o que demonstra grande interesse da indústria sobre o tema. 
Gráfico 1 - Patentes x Artigos

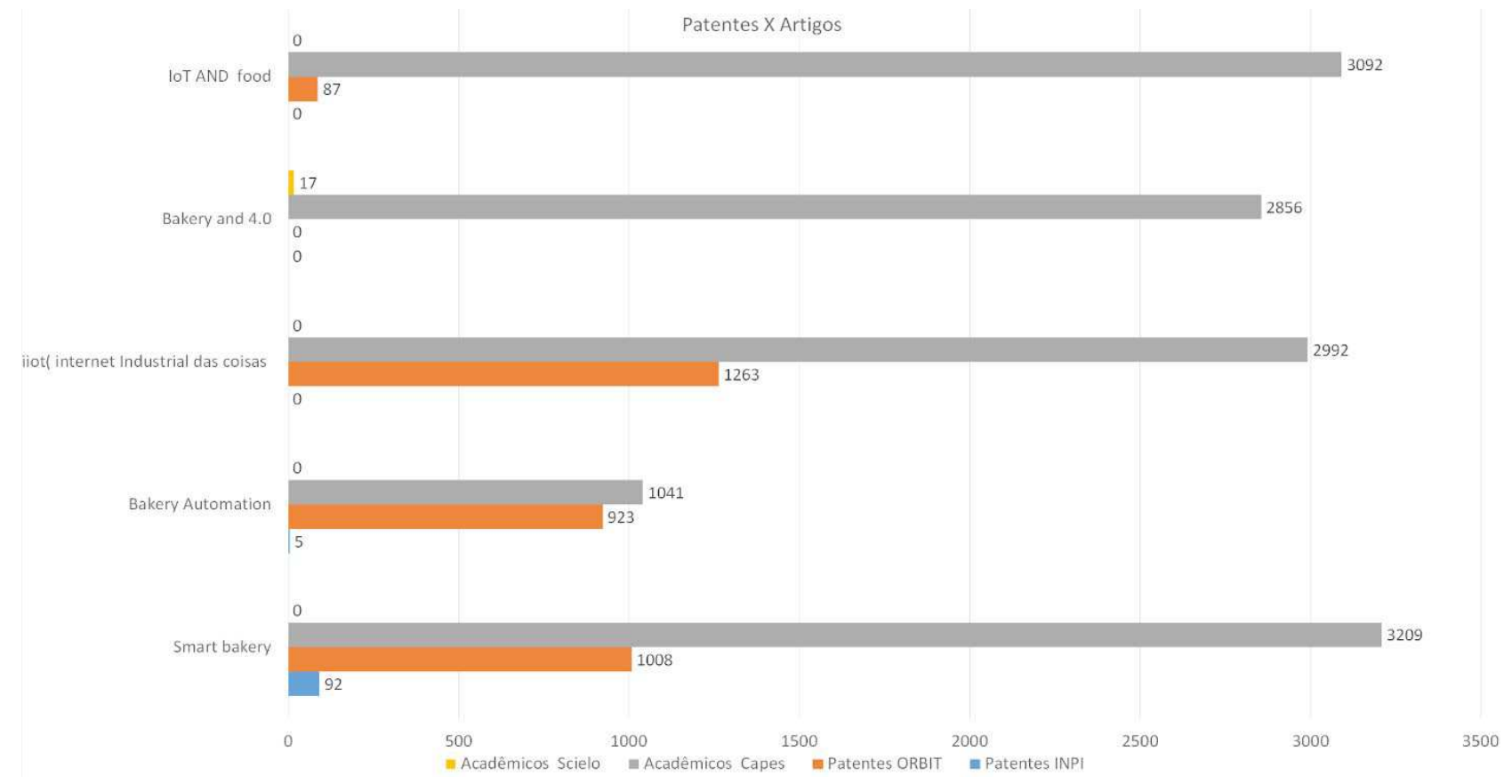

Fonte: Elaborado pelos autores deste artigo com dados da base Orbit Intelligence (2018)

$\mathrm{Na}$ análise dos principais depositantes de patentes a nível mundial, observa-se que as três empresas com maior número de patentes que se relacionam ao ramo alimentício voltado para a panificação são a Panasonic, Adeka e Fuji Oil e apenas uma instituição Universitária, a Voronezh State University da Rússia.

A Panasonic Corporation, empresa criada em 1918 e com atuação no Brasil desde 1967, apresenta maior número de patentes (691 unidades). A Panasonic investe em tecnologias ligadas a Bakery pelo fato de produzir diversos equipamentos destinados à produção de pães em casa, como os produtos Home Bakery e o Master Cheff Bread. Além disso, a empresa apresentou em 2017 na Consumer Electronic Show (CES) a maior feira de tecnologia do mundo em Las Vegas o "Robô Companheiro de Mesa" com vasta aplicação big data e Inteligência artificial (ESTADÃO, 2017).

Gráfico 2 - Principais depositantes -Termos de busca: bakery OR bread OR pastry OR confectionery OR (bake shop) OR (pastry shop) OR pâtisserie OR padaria OR panificação

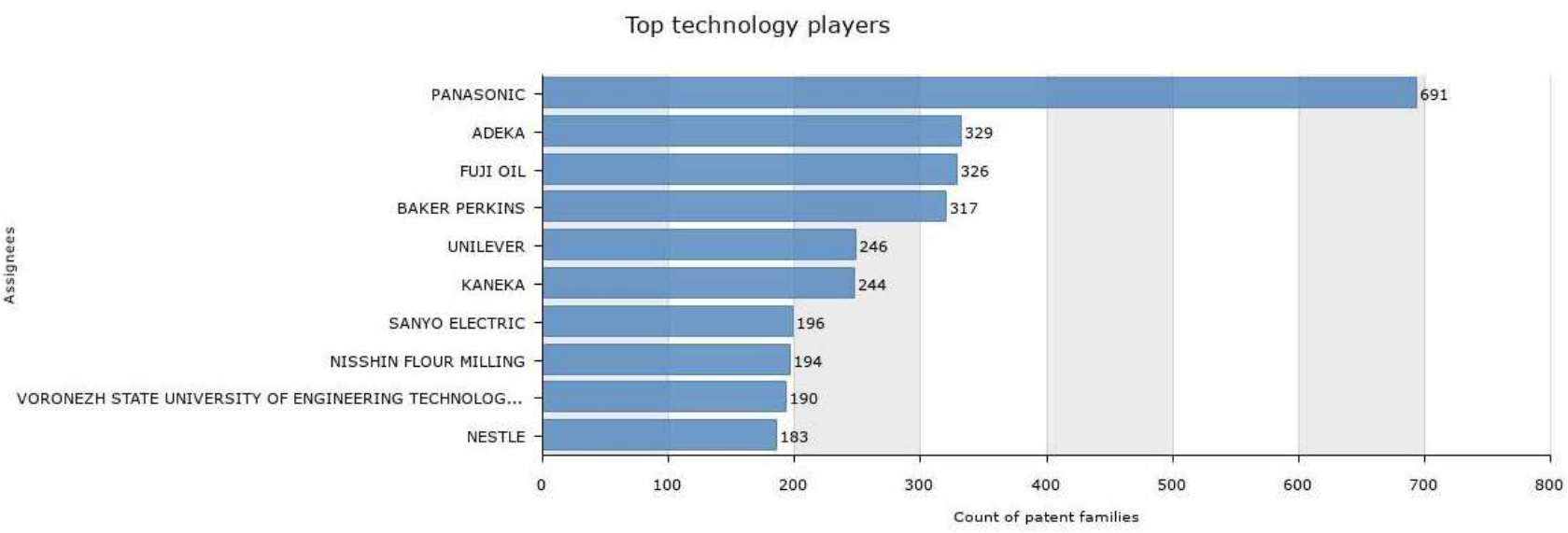

Fonte: Elaborado pelos autores deste artigo com dados da base Orbit Intelligence (2018) 
Utilizando os termos de buscas mais abrangentes (bakery OR bread OR pastry OR confectionery OR (bake shop) OR (pastry shop) OR pâtisserie OR padaria OR panificação), para o segmento de panificação, observou-se um aumento significativo nos depósitos de patentes no ano de 2016 (Gráfico 3). Tal fato pode ser atribuído ao desenvolvimento de tecnologias de integração em âmbito mundial.

Outro dado que pode explicar esse aumento expressivo no número de patentes se deve ao crescimento excepcional de pedidos de depósitos internacionais de patentes, de acordo com a World Intellectual Property Organization (WIPO, 2017), sendo mais de 3 milhões de depósitos de patentes registrados em 2016. Desse total, a Ásia apresentou 47,4\% dos pedidos via PCT, seguida pela Europa $(25,6 \%)$ e pela América do Norte (25,3\%). Observa-se, entretanto, que devido ao período de sigilo de 18 meses, os dados de 2017-2018 não estão completos. Levando em consideração a evolução da tecnologia, os resultados possivelmente continuarão em crescimento quando esses números forem computados.

Gráfico 3 - Evolução da tecnologia ao longo do tempo

Technology investment trend

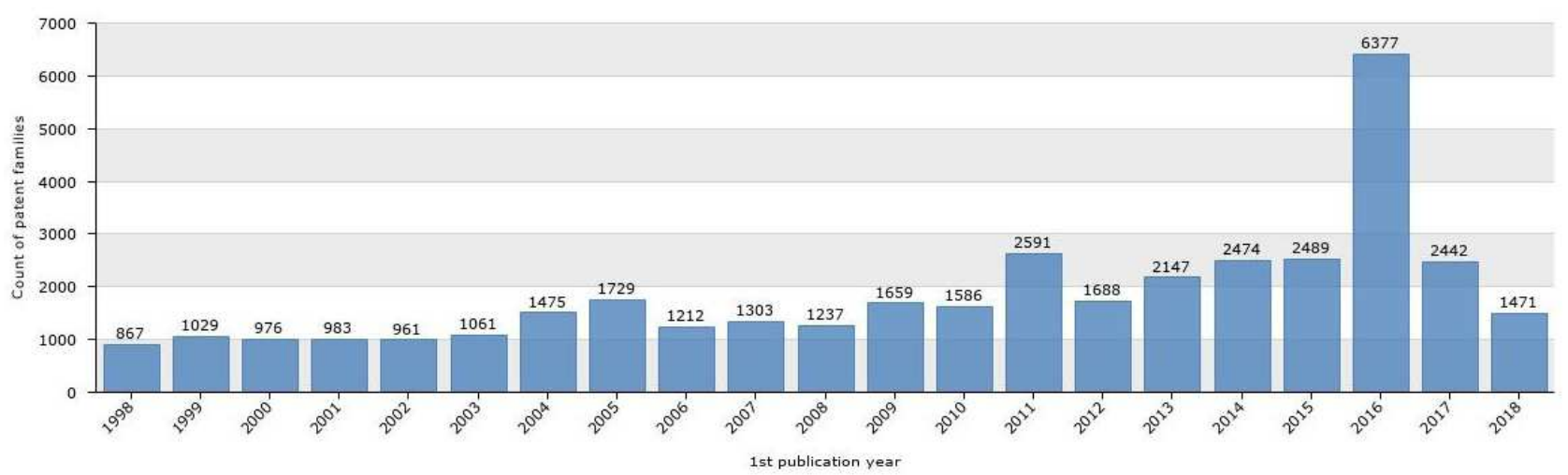

Fonte: Elaborado pelos autores deste artigo com dados da base Orbit Intelligence (2018)

Por meio da análise dos resultados das buscas com as palavras-chave, foram observados os códigos IPC de maior relevância: A21d-002/36 (Material Vegetal), A21d-013/00 (Produtos de panificação acabados ou semiacabados) e A21d-013/08 (Produtos multicamadas), conforme pode ser observado no Gráfico 4.

Vale ressaltar que entre as 10 classificações com maior número de patentes, nove são da categoria "A" e as três mais incidentes da subcategoria "A 21 D", sendo a seção "A": Necessidades Humanas, Subseção "21": cozedura ao forno; equipamento para preparo ou processamento de massas; massas para cozedura ao forno e "D": tratamento, por exemplo. Conservação de farinhas ou massas, por exemplo pela adição de materiais; cozimento; produtos de panificação; conservação dos produtos. 
Gráfico 4 - Patentes distribuídas por IPC (Termo de busca: bakery or bread or pastry or confectionery or (bake shop) or (pastry shop) or pâtisserie or padaria or panificação)

\section{Patent families by IPC codes}

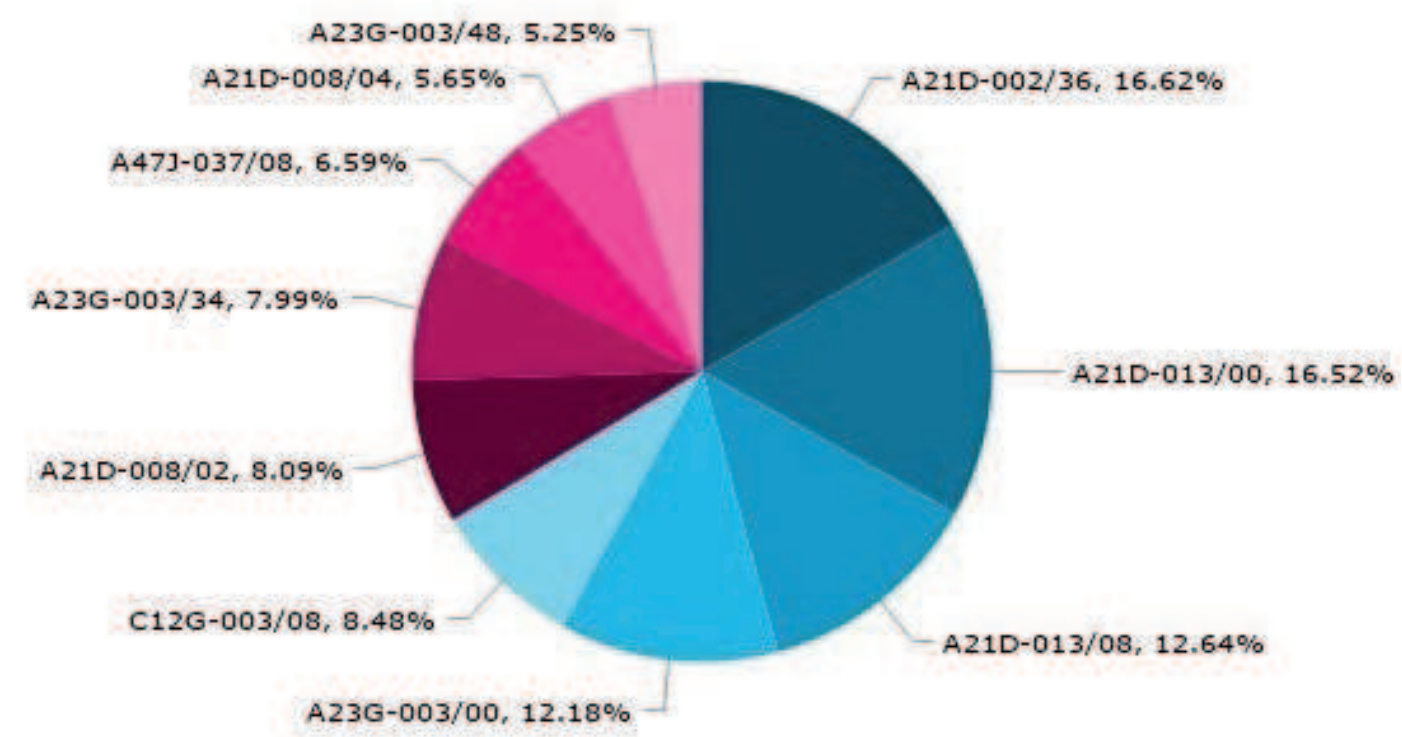

Fonte: Elaborado pelos autores deste artigo com dados da base Orbit Intelligence (2018)

Em relação à análise das patentes relacionadas diretamente ao termo "IoT AND Food", observou-se que a predominância das classificações IPC mudou para a categoria "G", que corresponde à seção da Física, a qual se dividiu em outras subcategorias como a G05B: sistemas de controle ou regulagem em geral; elementos funcionais de tais sistemas; disposições para monitoração ou teste de tais sistemas ou elementos; e a G06Q: sistemas ou métodos de processamento de dados, especialmente adaptados para propósitos administrativos, comerciais, financeiros, de gerenciamento, supervisão ou predição; sistemas ou métodos especialmente adaptados para propósitos administrativos, comerciais, financeiros, de gerenciamento, supervisão ou predição, não incluídos em outro local.

Gráfico 5 - Patentes distribuídas pelo IPC (IoT AND food)

Patent families by IPC codes

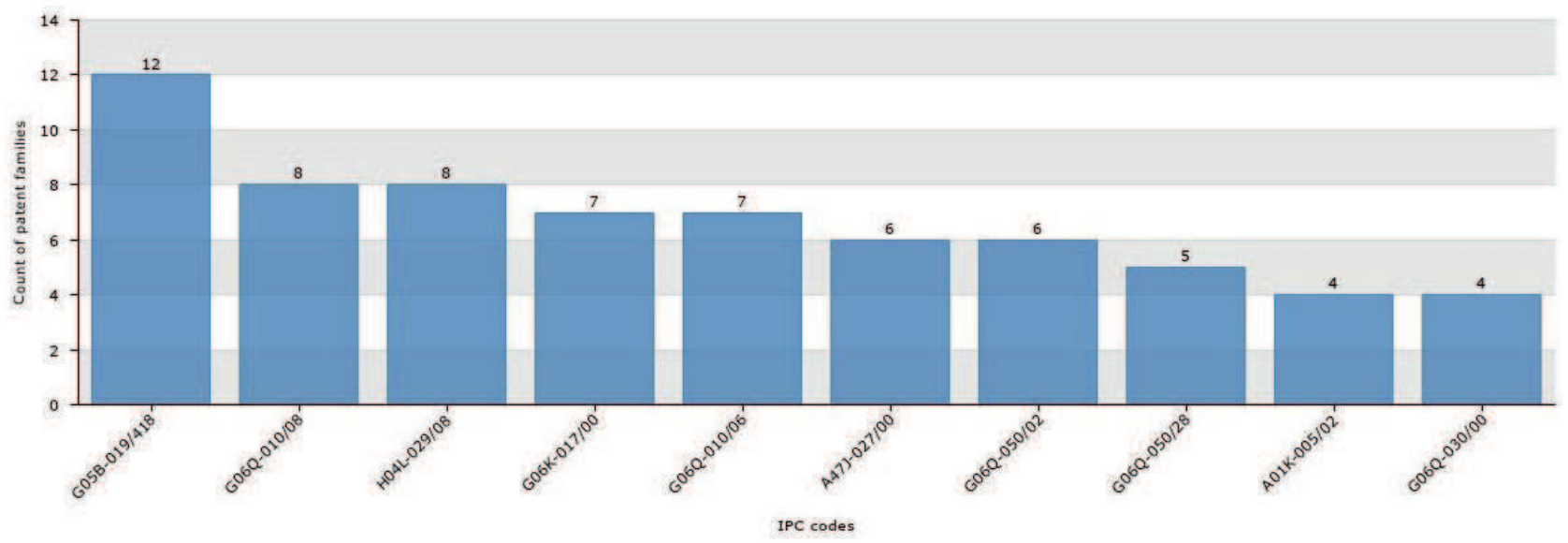

Fonte: Elaborado pelos autores deste artigo com dados da base Orbit Intelligence (2018) 
O Gráfico 6 revelou que a produção de patentes está sendo direcionada para três vertentes na gestão dos negócios: métodos administrativos, controle e comunicação digital. Essas linhas de aplicação apresentam potencial de transformação significativo para o desenvolvimento de modelos de negócios automatizados, cortando custos, aumentando a produtividade e atingindo um maior número de clientes por meio de recursos tecnológicos de interoperabilidade.

Gráfico 6 - Visão Geral da tecnologia (IoT AND food)

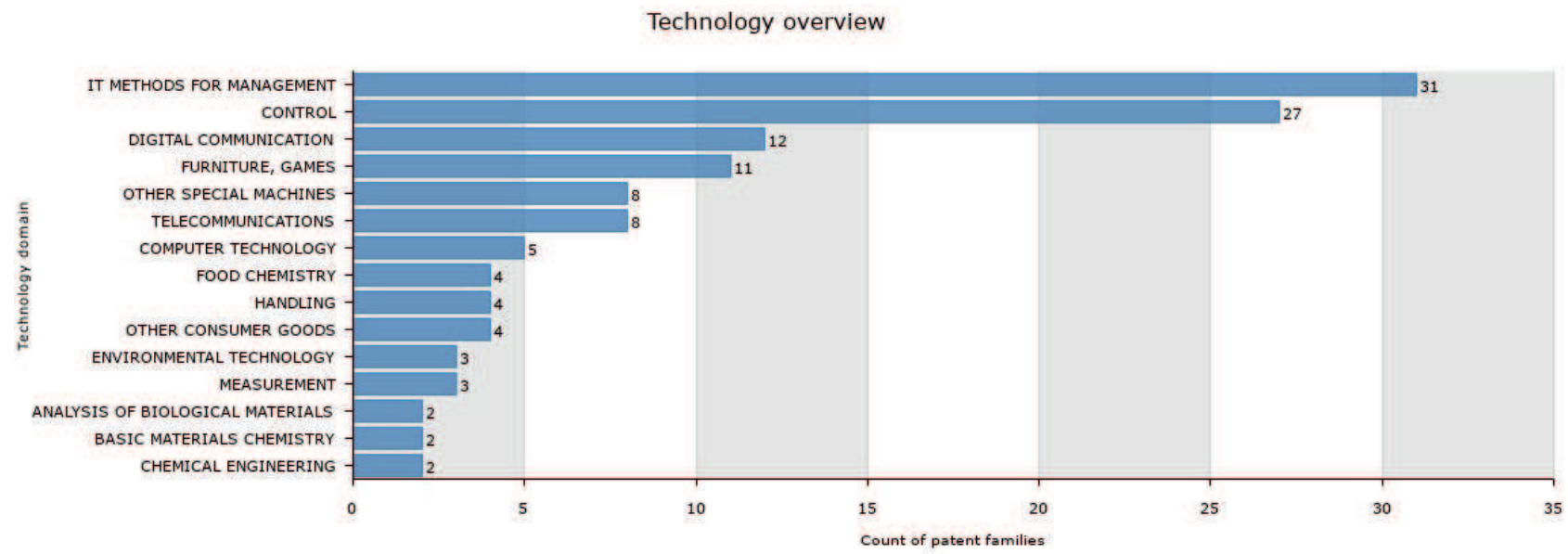

Fonte: Elaborado pelos autores deste artigo com dados da base Orbit Intelligence (2018)

Observou-se no Gráfico 7 que essa tecnologia é recente, pois apresenta apenas oito anos. Apesar disso, foi observada sua importância mercadológica e, em 2017, ocorreu um crescimento significativo em depósitos de patentes. Constatou-se, ainda, que devido ao período de sigilo, esses números provavelmente continuarão em crescimento.

Gráfico 7 - Investimento na tecnologia ao longo do tempo (IoT AND food)

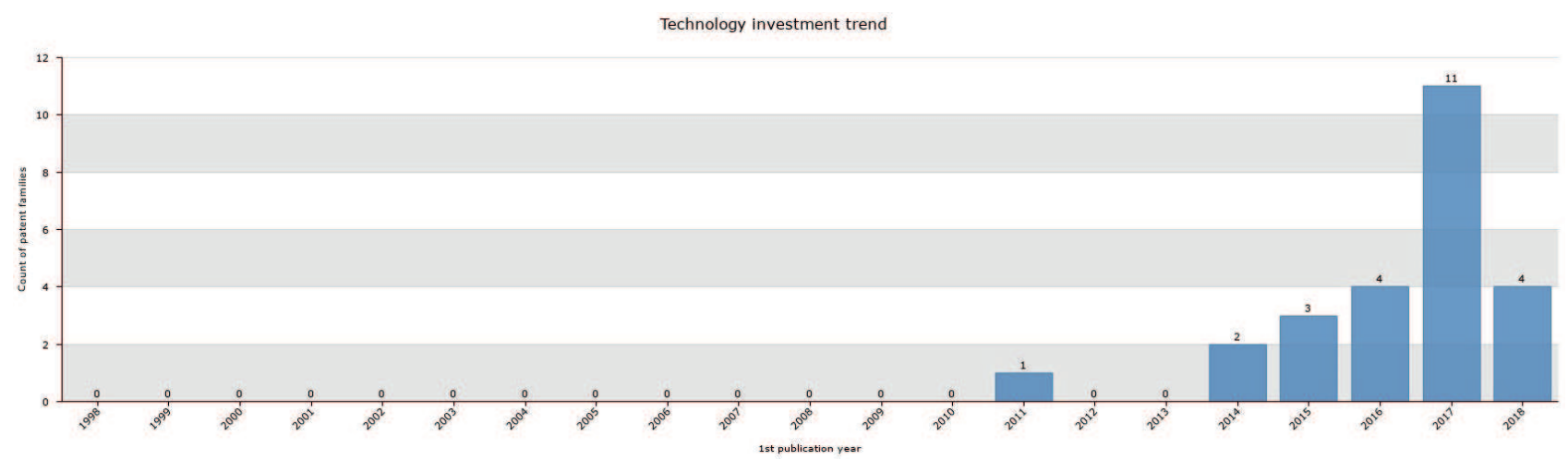

Fonte: Elaborado pelos autores deste artigo com dados da base Orbit Intelligence (2018)

Por meio das informações extraídas da Plataforma Orbit observou-se a predominância da China em relação aos depósitos de patentes desse segmento. Esse é um dado relevante, pois além de possuir a maior população mundial; a China é um dos países que mais produz e exporta produtos com tecnologia de ponta. Observa-se que, em paralelo, outros países como Brasil, Canadá, EUA, Austrália e Índia também desenvolvem pesquisas relacionadas com o ramo alimentício e a Internet das Coisas, porém em menor proporção. 
Figura 2 - Mercados e localização competitiva (IoT AND food)

Markets \& competitors location

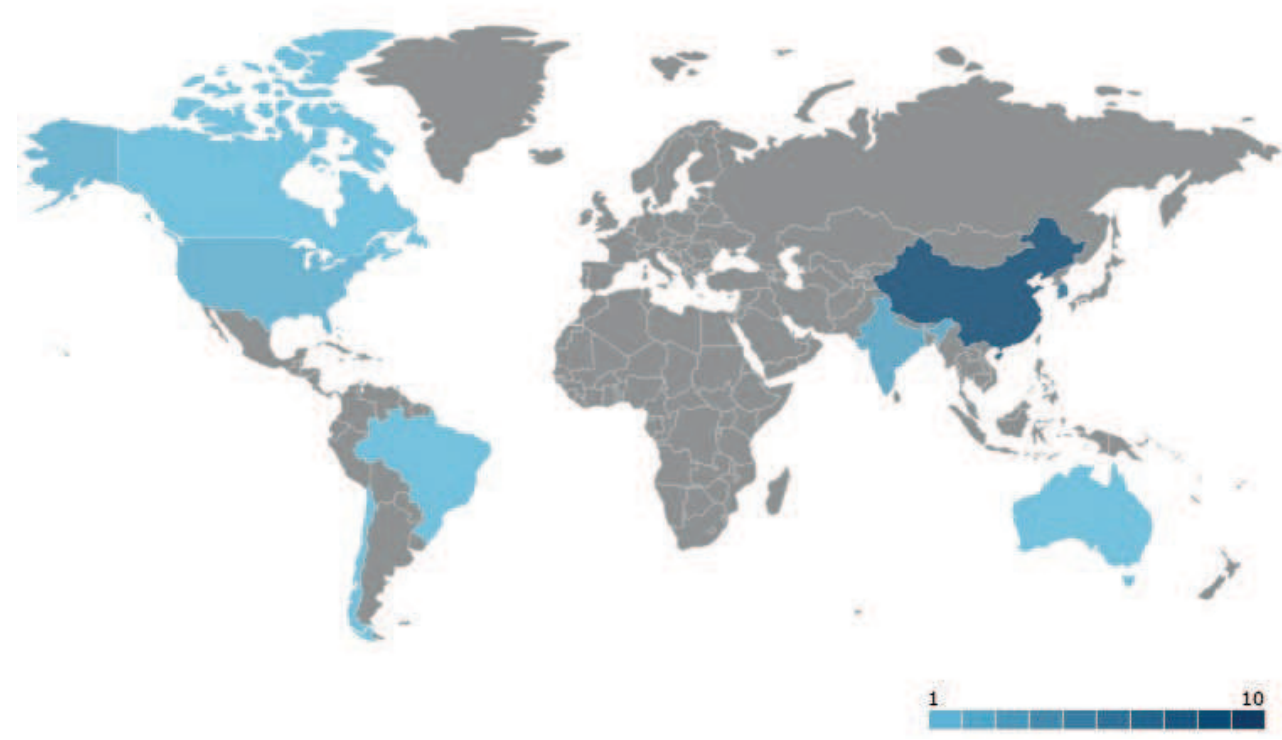

Fonte: Elaborada pelos autores deste artigo com dados da base Orbit Intelligence (2018)

Na busca pelos termos "IoT AND Food" na plataforma Google Trends, constatou-se uma tendência de crescimento de busca pelo tema no mundo, porém, quando o recorte é feito no Brasil (adaptando-se os termos de buscas para o português), o resultado é nulo, ou seja, ainda não há dados estatísticos de busca desses termos no Brasil.

Gráfico 8 - Interesse ao longo do tempo Google Trends pelo termo IoT and Food

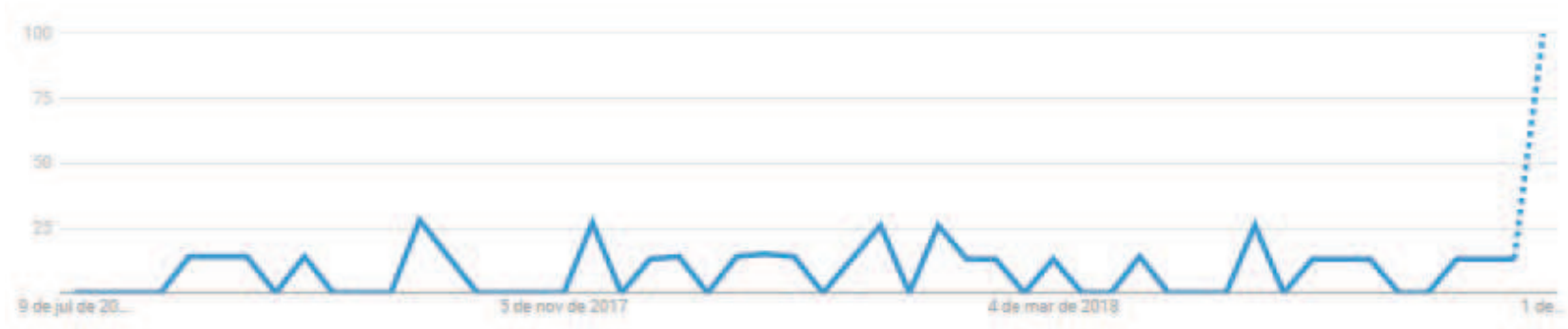

Fonte: Elaborado pelos autores deste artigo com o uso da plataforma Google Trends

\section{Considerações Finais}

A realização desta pesquisa permitiu a verificação do panorama mundial dos estudos e das tecnologias desenvolvidas que apresentam relação com a Internet das Coisas (IoT) e com os equipamentos utilizados em panificação e confeitaria. As prospecções realizadas revelam que a China é o país com maior número de patentes do segmento, enquanto o Brasil apresenta um cenário ainda incipiente para equipamentos inteligentes com potencial de transformar de forma significativa as formas de venda e de consumo de produtos oferecidos por panificações e confeitarias. 
O desenvolvimento de equipamentos com capacidade de estabelecimento de comunicação entre si é uma tendência mundial e os países vêm buscando criar cenários que favoreçam a absorção e o desenvolvimento dessas tecnologias. Esses dados apontam para a necessidade de maior monitoramento $e$ de investimento em pesquisas e desenvolvimento de tecnologias inteligentes de automação voltadas para empresas de alimentos, nesse caso, especificamente as empresas de panificação e de confeitaria, desenvolvendo-se ativos que venham a suprir uma tendência global da indústria 4.0.

Verifica-se ainda que, apesar do baixo índice de inovação no Brasil, há um movimento nacional de incentivo à criação de ambientes propícios para o desenvolvimento de produtos que apresentem interação por meio da tecnologia IoT, com a criação de leis, normas e programas de incentivo. Com o lançamento em 2018 do Programa Nacional de Internet das Coisas, espera-se a promoção de tecnologias inteligentes que também possam atender a esse ramo $e$ viabilizar novos estudos.

\section{Referências}

BIAGGI, G. Q. et al. A internet das coisas como fator primordial na indústria 4.0. Revista Eletrônica Engenharia Estudos e Debates, [S.I.], v. 1, 2018.

BODLA, R. Internet of Things: The Key Ingredient in Next-Generation Food and Beverage Companies. Revista Food Manufacturing, [S.l.], 2015. Disponível em: <https://www. foodmanufacturing.com/article/2015/11/internet-things-key-ingredient-next-generation-food-andbeverage-companies >. Acesso em: 20 jun. 2018.

ESTADÃO. Panasonic demonstra robô “Companion” na CES. jan. 2017. Disponível em: $<$ https://economia.estadao.com.br/noticias/releases-ae, panasonic-demonstra-robo-companion-naces-2017,10000098301>. Acesso em: 30 jun. 2018.

FIRJAM - FEDERAÇÃO DAS INDÚSTRIAS DO ESTADO DO RIO DE JANEIRO. Indústria 4.0. Cadernos SENAI de Inovação, [S.l.], 2016.

REVISTA FORBES. 5 Easy To Understand Examples Of The Internet of Things. jan, 2016. Disponível em: <https:/www.forbes.com/sites/blakemorgan/2016/01/27/5-easy-to-understandexamples-of-iot-and-customer-experience/\#62a10311366c>. Acesso em: 20 jun. 2018.

MARTINEZ, M. E. M.; SANTOS, D. A.; WINTER, E. Mapeamento por meio de documentos patentários depositados no Brasil das tecnologias do setor de celulose e papel. O Papel, [S.l.], v. 76, n. 10, p. 77-81, 2015.

MINISTÉRIO DA CIÊNCIA, TECNOLOGIA, INOVAÇÕES E COMUNICAÇÕES (MCTIC). MCTIC e BNDES apresentam estudo do Plano Nacional de IoT com 76 açóes para o setor, 2017. Disponível em: < http://www.mctic.gov.br/mctic/opencms/salalmprensa/noticias/arquivos/2017/10/ MCTIC_e_BNDES_apresentam_estudo_do_Plano_Nacional_de_IoT_com_76_acoes_para_o_setor. html>. Acesso em: 29 jun. 2018

PEREIRA, A.; SIMONETTO, E. O. Indústria 4.0: conceitos e perspectivas para o Brasil. Revista da Universidade Vale do Rio Verde, [S.I.], v. 16, n. 1, 2018.

SANTAELLA, L. et al. Desvelando a internet das coisas. Revista GEMInIS, [S.I.], v. 4, n. 2, p. 19$32,2013$. 
SEBRAE - SERVIÇO BRASILEIRO DE APOIO ÀS MICRO E PEQUENAS EMPRESAS. Tecnologia e Inovação na Panificação e Confeitaria, 2016. Disponível em: <http://www.abip.org.br/ encarteinovacaoetecnologia.pdf. >. Acesso em: 19 jun. 2018.

ZAMBARDA, P. Internet das Coisas: entenda o conceito e o que muda com a tecnologia. Tech tudo. 2014. Disponível em: <http://www.techtudo.com.br/noticias/noticia/2014/08/internet-dascoisas-entenda-o-conceito-e-o-que-muda-com-tecnologia.html>. Acesso em: $1^{\circ}$ jul. 2018.

WIPO - WORD INTELLECTUAL PROPERTY ORGANIZATION. Record Year for International

Patent Applications. 2017. Disponível em: <http://www.wipo.int/pressroom/en/articles/2017/ article_0002.html.>. Acesso em: 29 jun. 2018.

\section{Sobre os autores}

\section{Beneildo Rodrigues Oliveira Pereira}

E-mail: beneildop@hotmail.com

Mestrando pelo Programa de Pós-graduação em Propriedade Intelectual e Transferência de Tecnologia para a Inovação (PROFNIT) (2018). Especialista em Gestão Pública pela Faculdade Integrada de Jacarepaguá, RJ.

Endereço profissional: Instituto de Química e Biotecnologia (IQB), Universidade Federal de Alagoas (UFAL). Av. Lourival de Melo Mota, s/n, Tabuleiro do Martins, Maceió, AL. CEP: 57072-970.

\section{Clodoaldo Silva do Nascimento}

E-mail: clodoaldo.al@hotmail.com

Mestrando pelo Programa de Pós-graduação em Propriedade Intelectual e Transferência de Tecnologia para a Inovação (PROFNIT) (2018). Graduado em Administração pelo Centro Universitário Cesmac.

Endereço profissional: Universidade Federal de Alagoas (UFAL). Av. Presidente Getúlio Vargas, n. 50, Serraria, Maceió, AL. CEP: 57046-140.

\section{Jéssica Caroline Rodrigues de Lima}

E-mail: jessi.rodrigues90@hotmail.com

Mestranda em Arquitetura e Urbanismo - Dinâmicas do Espaço Habitado da Universidade Federal de Alagoas (UFAL). Graduada em Arquitetura e Urbanismo pela Universidade Federal de Alagoas (2015).

Endereço profissional: Faculdade de Arquitetura e Urbanismo, Universidade Federal de Alagoas (UFAL). Av. Lourival Melo Mota, s/n, Tabuleiro do Martins, Maceió, AL. CEP: 57072-900.

\section{João Paulo Lima Santos}

E-mail: jpls@lccv.ufal.br

Doutor em Engenharia Civil pela Universidade Federal do Rio de Janeiro (2011). Mestre em Engenharia Civil pela Universidade Federal do Rio de Janeiro (2008).

Endereço profissional: Universidade Federal de Alagoas (UFAL). Av. Lourival de Melo Mota, s/n, Tabuleiro do Martins, Maceió, AL. CEP: 57072-970.

\section{Ramon Cardeal Silva}

E-mail: ramoncardeal@gmail.com

Mestrando pelo Programa de Pós-Graduação em Propriedade Intelectual e Transferência de Tecnologia para a Inovação PROFNIT (2018). Graduado em Administração pela Universidade Federal de Alagoas (2014).

Endereço profissional: Universidade Federal de Alagoas (UFAL). Av. Lourival de Melo Mota, s/n, Tabuleiro do Martins, Maceió, AL. CEP: 57072-970. 


\section{Silvia Beatriz Beger Uchôa}

E-mail: sbuchoa@ctec.ufal.br

Doutora em Química e Biotecnologia - Área de concentração Físico-Química pelo Instituto de Química e Biotecnologia da UFAL (2007). Mestre em Arquitetura e Planejamento, pela Escola de Engenharia de São Carlos da Universidade de São Paulo (1989).

Endereço profissional: Universidade Federal de Alagoas (UFAL). Av. Lourival de Melo Mota, s/n, Tabuleiro do Martins, Maceió, AL. CEP: 57072-970. 